\title{
ALGUNAS OBSERVACIONES SOBRE EL USO DEL SUELO EN EL SITIO ARQUEOLÓGICO DE CALAKMUL' ${ }^{1}$
}

\section{William J. Folan y Silverio Gallegos Osuna}

Centro de Investigaciones Históricas y Sociales-unc

\begin{abstract}
Aвstrucr: During the past 50 years, a series of investigators has examined bajos (seasonal swamps) in the development of Maya civilization, especially in the Peten of Campeche, Mexico, and Guatemala where a great deal of the landscape is formed by these depressions. They have been the bane of travelers especially during the rainy season, when their fooded bottoms turn into sucking quagmires capable of stopping man, beast, or machine. Several investigators, such as Richard E. W. Adams, have suggested that bajos were used to develop raised fields. In some cases the edges were dedicated to slash and burn horticulture. This paper presents a four stage model of Maya horticulture developed by Joel D. Gunn and ourselves. It includes milpa horticulture located on upland plots and slopes, as well as those on the plain following the bajo-edge. Horticulture also takes place within the bajo on small plots of earth protruding from the floor close to its edge. They are planted during the regular rainy season and following it in the form a tonamil later planted into the bajo floor to supplement an October harvest. Later work by Jacinto May Hau (while searching for the long-distances sacbes of Calakmul in the Laberinto Bajo) has discovered large, three to four hectare plots of land in the bajo limited by $40-60 \mathrm{~cm}$ high albarradas (dry stone walls). They are situated on elevated ground within the bajo to facilitate intensive agricultural activities. This suggest that although there may or may not exist raised fields in the sense suggested by Adams in the Peten or Richard Hansen in Nakbe, Guatemala, bajos in general may well have been utilized for agricultural and horticultural purposes in one manner or another.
\end{abstract}

Resumev: A través de los siglos los habitantes del área maya han sido conscientes de sus fuentes hídricas, de modo que han buscado diferentes formas para captar este elemento tan indispensable para su sobrevivencia o dirigirlo hacia determinado lugar cuando se presenta en cantidades inadecuadas o excesivas a sus necesidades. Sin embargo, un interrogante que tendrá que responderse es durante qué periodos de su desarrollo los mayas estuvieron en posición de utilizar ciertas técnicas agrícolas y hortícolas en cualquier región de su territorio donde, según el modelo considerado por varios investigadores, el acceso a la humedad, en términos generales, variaba de tiempo en tiempo y de lugar a lugar en toda la región de Mesoamérica, todo ello relacionado en parte con el régimen de precipitación.

Calakmul, localizado en el sureste del estado de Campeche, es uno de los sitios prehispánicos más grandes de Mesoamérica (Folan, 1985a; Marcus, Pincemin, Dominguez, Fletcher y Morales, 1995)

\footnotetext{
${ }^{1}$ Este documento representa una ponencia revisada, que antes fue leída como parte del simposio organizado por la Universidad de California, Riverside, Camellones Tropicales, llevado a cabo en Villahermosa, Tabasco, en 1991. Estudios de Cultura Maya. Vol. XXI, 2000

Instituto de Investigaciones Filológicas/

Centro de Estudios Mayas, UNAM ISSN: 0185-2574
} 


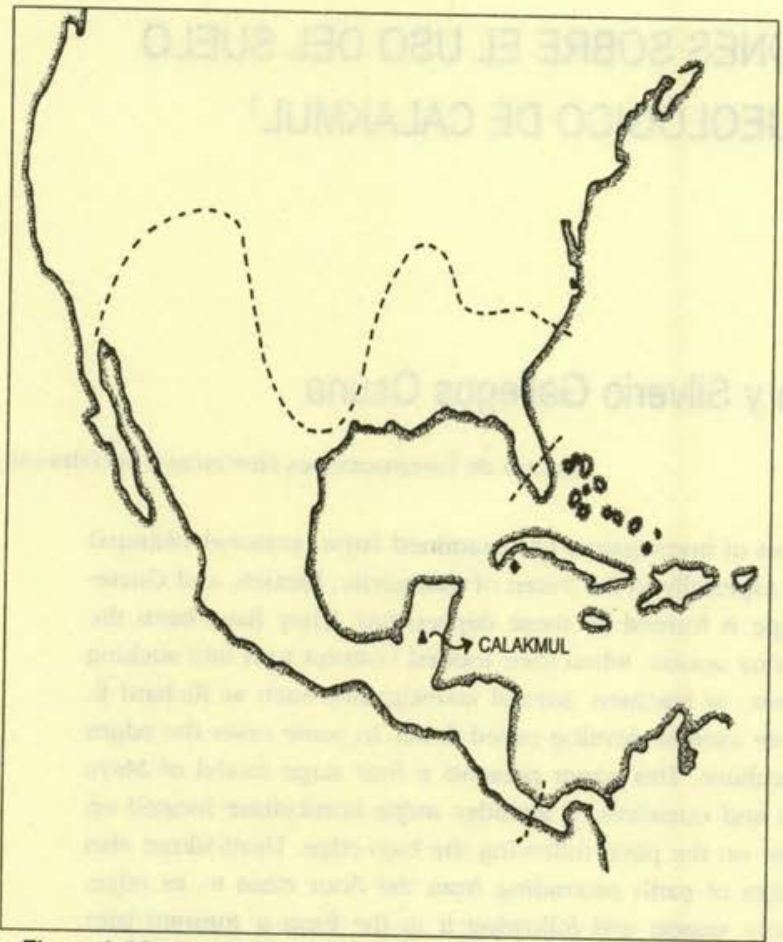

Figura 1. Mapa que muestra la ubicación de Calakmul en la Gran Mesoamérica (dibujo de Juan José Cosgaya Medina)

que Calakmul fue un sitio de gran importancia en Mesoamérica durante el inicio de la era cristiana. Esto se manifiesta parcialmente por la presencia de la estructura II, que mide entre $50 \mathrm{y}$ $55 \mathrm{~m}$ de altura por $140 \times 140 \mathrm{~m}$ en su base, asemejándose a la estructura de El Tigre, dentro de lo que he llamado la ciudad gemela de El Mirador, localizada a unos $37 \mathrm{~km}$ al suroeste de Calakmul en la región norte del Petén de Guatemala (Folan, 1985b, 1988; Hansen, 1994). Además de sus grandes atributos arquitectónicos, las excavaciones de Calakmul -realizadas por el Centro de Investigaciones Históricas y Sociales (CIHS) de la Universidad Autónoma de Campeche (UAC), en colaboración con el estado de Campeche, el Instituto Nacional de Antropología e Historia, el Pider, el Instituto Nacional Indigenista, la Digesca y la National Geographic Society - han mostrado la importancia de este sitio en

(figura 1). Ya se ha hecho un mapa de $30 \mathrm{~km}^{2}$ de este importante centro con sus 6250 edificios y otros rasgos materiales, con ayuda de $360 \mathrm{~km}$ de brechas y durante 84 meses de trabajo en el campo; el área del mapa equivale aproximadamente a la actual ciudad preindustrial de Campeche (Folan, May Hau, Cohouh Muñoz y González Heredia, 1990). El asentamiento de Calakmul se extiende tanto al norte como al sur y este del área incluida en nuestro mapa (figura 2). Además de ser una ciudad planificada en forma concéntrica, Calakmul también es lacustre y sigue la orilla del bajo de El Laberinto, de unos $24 \mathrm{~km}$ de longitud a lo largo de una gran distancia (Lundell, 1933) y tal vez continúe extendiéndose hacia el otro lado (Fletcher et al., 1987) comunicado por sacbés (Folan, Marcus y Miller, 1995).

Hasta ahora nuestras excavaciones indican Estudios de Cultura Maya. Vol. XXI, 2000 Instituto de Investigaciones Filológicas/ Centro de Estudios Mayas, UNAM ISSN: 0185-2574 el pasado, manifestada parcialmente por el descubrimiento de tres tumbas reales de los siglos v y viII. La primera de estas tumbas fue excavada en la estructura VII del sitio por Lagunas (1985) y Domínguez y Gallegos (1989-1990). Su entrada está localizada en medio de la primera de sus tres crujias. Esta tumba representa la última morada de un gobernante de Calakmul, que fue enterrado con su capa de piel de jaguar y unas 2220 piezas de jade. Todas fueron trabajadas -incluso las piezas más pequeñas- hasta lograr crear una máscara retrato del personaje formada por placas de jade, reconstruida por el maestro Jaime Camas del inaH. La otra tumba real fue excavada por debajo del cuarto localizado en medio del edificio III de Calakmul, también conocido como el Palacio Lundell (Coyoc Ramírez, 1989 y Pincemin Deliberos, 1989. 1994). Esta gran tumba, con su bóveda maya y 


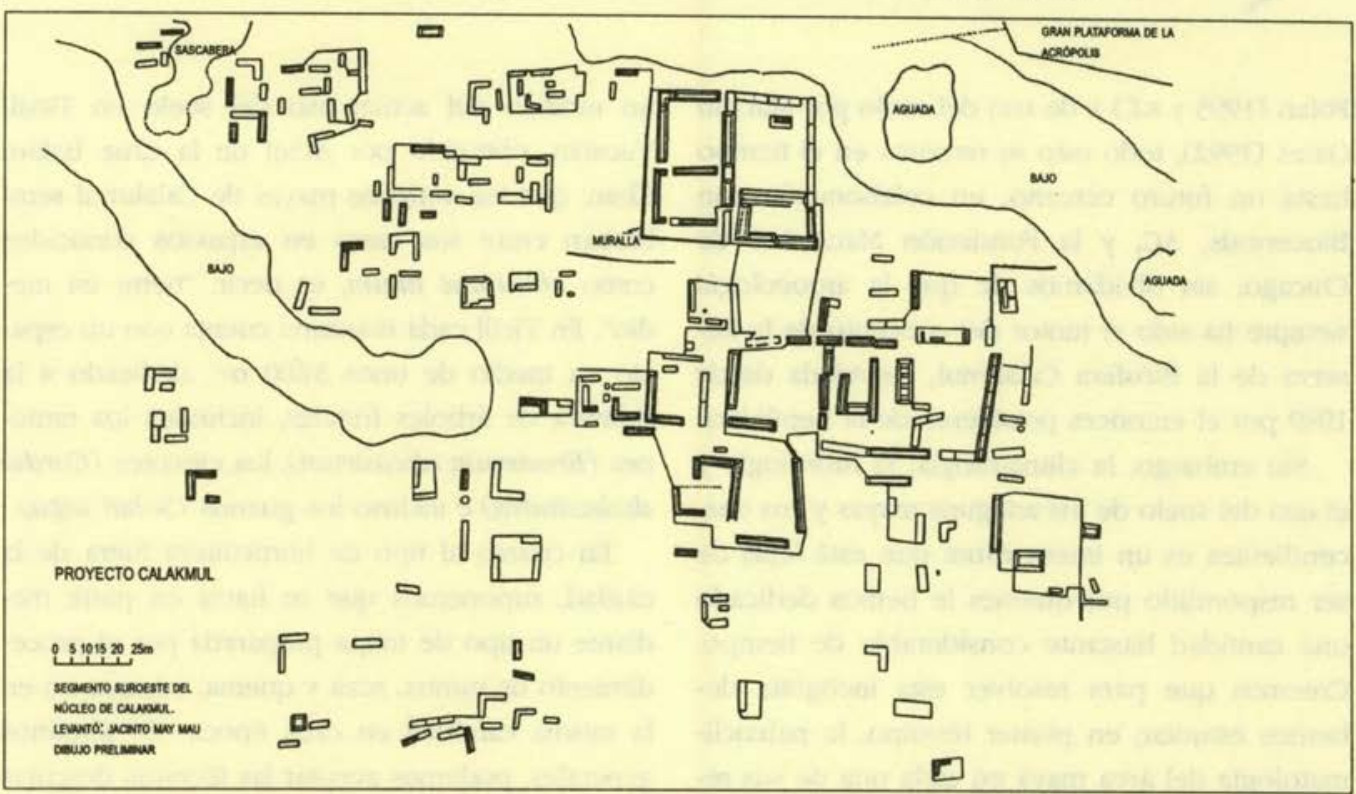

FuentE: William J. Folan y Román Piña Chan, "El Proyecto Calakmul", en Información, 3-4, Centro de Estudios Históricos y Sociales, Universidad Autónoma del Sudeste, Campeche, abril-diciembre de 1982, pp. 24-46 (el mapa está en la última página).

un ducto al exterior de la estructura, también perteneció a un gobernante de mediana edad acompañado por una elegante máscara retrato formada de mosaicos de jade y concha. Igualmente había dos máscaras hechas con mosaicos de jade, una de ellas encontrada a la altura del tórax y otra alrededor de la cintura, todas restauradas por García Cruz y Pérez Herrera (1989), ambos colaboradores del inaH. Además de las máscaras y cuentas de jade, la tumba tenía tres placas de este mismo material que nos proporcionarian el glifo de su ocupante, según Marcus (1989). También acompañaban al difunto varias vasijas, incluida una tetrápoda con rostro, y comida adentro, y otra policromada que presenta figuras de serpientes estilizadas, tal vez con una preparación de cacao en su interior como en Río Azul, Guatemala (Adams, 1986). La última tumba, excavada por el arqueólogo Abel Morales López (1994, y Folan y Morales López, 1996), contiene otra máscara de mosaicos, un pendiente del Dios Descendente y una vasija tipo códice.

Estudios de Cultura Maya. Vol. XXI, 2000 Instituto de Investigaciones Filológicas/ Centro de Estudios Mayas, UNAM ISSN: 0185-2574
Aunque las excavaciones en Calakmul han producido descubrimientos de varias clases, una de las hazañas más sobresalientes del Proyecto Calakmul, en colaboración con la Sedue, hoy Secretaría del Medio Ambiente, Recursos Naturales y Pesca, y el inaH, fue la formación de la Reserva de la Biósfera de Calakmul, de aproximadamente 723000 ha (Folan, 1978, 1984), incluyendo casi totalmente la región prehispánica de Calakmul durante su periodo Clásico, que comprende sitios tributarios ya desarrollados en un modelo por Flannery (1972) y Marcus (1976), además de su ambiente natural (figura 3 ).

Aparte de los trabajos llevados a cabo entre la Universidad y la Sedue (Folan, García Ortega y Sánchez González, 1992) para formular un programa de manejo para la reserva, más los trabajos sobre su flora, fauna y geografia, hechos por nuestros colaboradores, el cris ha estado realizando investigaciones sobre la climatología de la reserva por parte de Joel Gunn et al. (1994 y 1995), de hidrología por Gary Gates y 
Folan (1993 y s.f.) y de uso del suelo por Marylin Gates (1992), todo esto se remonta en el tiempo hasta un futuro cercano, en colaboración con Biocenosis, AC, y la Fundación MacArthur de Chicago, sin olvidarnos de que la arqueología siempre ha sido el motor del concepto de la Reserva de la Biósfera Calakmul, decretada desde 1989 por el entonces presidente de la República.

Sin embargo, la climatología, la hidrología y el uso del suelo de los antiguos mayas y sus descendientes es un interrogante que está lejos de ser respondido por quienes le hemos dedicado una cantidad bastante considerable de tiempo. Creemos que para resolver esta incógnita debemos estudiar, en primer término, la paleoclimatología del área maya en cada una de sus regiones, en combinación con la paleohidrología y la oceanografia o de lo contrario no llegaremos a soluciones lógicas (Folan et al., 1983; Gunn y Folan, 1995; Gunn, Folan y Robichaux, 1994, 1995). Por ejemplo, no podemos suponer que los mayas del Petén de Campeche y Guatemala hayan llevado a cabo la misma tecnología agrícola que los mayas del área ribereña de $\mathrm{Be}$ lice en épocas similares o diferentes de precipitación, o bien que los mayas de la región Puuc hayan obtenido los mismos beneficios o perjuicios con sus siembras que los habitantes de la parte norte de la península, asentados donde existían cenotes y con un régimen climático de cierta variación. De alguna manera hay que encontrar la relación diacrónica entre climatología, hidrología y técnicas hortícolas para comprender los beneficios y problemas hallados en determinada región, asociada con grupos de gente de lengua maya en todas las épocas.

Con respecto al uso del suelo y a la hidrología, los antiguos mayas conocían diversas técnicas, inclusive el sistema propuesto por Netting (1977) de formar zonas hortícolas dentro de sus áreas de habitación y campos de cultivo en las

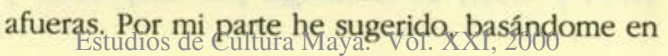
Instituto de Investigaciones Filológicas/

Centro de Estudios Mayas, UNAM ISSN: 0185-2574 un modelo del actual uso del suelo en Ticul, Yucatán, obtenido por Adiel de la Cruz Balam Chan, que los antiguos mayas de Calakmul sembraban entre sus casas en espacios conocidos como chuumuc lu'um, es decir, "tierra en medio". En Ticul cada manzana cuenta con un espacio en medio de unos $3600 \mathrm{~m}^{2}$, dedicado a la siembra de árboles frutales, incluidos los ramones (Brosimum alicastrum), los ciricotes (Cordia dodecandra) e incluso los guanos (Sabal yapa).

En cuanto al tipo de horticultura fuera de la ciudad, suponemos que se hacía en parte mediante un tipo de milpa preparada por el procedimiento de tumba, roza y quema, aunque no en la misma cantidad en cada época. En términos generales, podemos aceptar las técnicas descritas por Silverio Gallegos Osuna, un joven milpero de la región, quien escribe acerca de cómo los antiguos podrían haber cultivado la milpa alrededor de los bajos, durante por lo menos una parte de su existencia en Calakmul (figura 4). Según este autor, los milperos ubicados alrededor de Conhuas, Campeche, y tal vez de Calakmul, hacían la milpa tanto en la falda como en la orilla del bajo, porque es en la falda donde se encuentra más tierra yaxbom, o sea kankab, y donde se puede sembrar hasta por cuatro años continuos, como sucede con una milpa hechà dentro de un ramonal. Esta misma milpa se extiende dentro del bajo donde se encuentran pequeñas elevaciones (cuyitos) aparentemente naturales, de unos $25 \times 25 \times 25 \mathrm{~cm}$ y algunas más grandes, tal vez creadas por el hombre y la naturaleza, de 1 a $2 \mathrm{~m}$ de longitud por 40 a $70 \mathrm{~cm}$ de ancho y $40 \mathrm{~cm}$ de altura en forma de cacahuate. Al tiempo de sembrar parte de la milpa, en la falda del bajo, donde hay bastante pedernal, los milperos también siembran los cuyos chicos y grandes que encuentran en sus milpas dentro del bajo, en cantidades de 40 a 50 aproximadamente por mecate de $25 \times 25 \mathrm{~m}$, o sea $625 \mathrm{~m}^{2}$. Ambos cuyos, chicos y grandes, protegen el maíz dentro del 


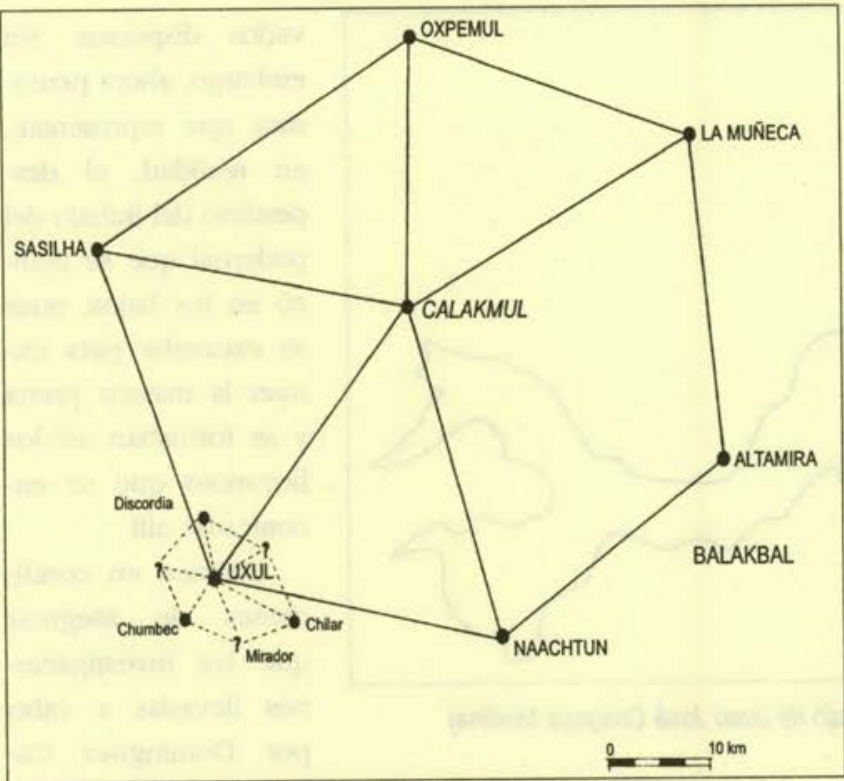

Figura 3. El Estado regional de Campeche y sus ciudades tributarias (Marcus, 1976)

bajo si éste se llena con una cantidad razonable de agua. En el mes de octubre, cuando el maíz sembrado en mayo está listo para cosecharse, si la cosecha no satisface sus necesidades, el milpero puede decidir sembrar un tonamil o segunda siembra, pero más profunda que la primera en la parte de la milpa entre los cuyos pequeños en el bajo, si el bajo no está muy encharcado, practicando así una variante de agricultura tipo llano inundado o flood plain agriculture.

Además de cuyos pequeños, hay algunos grandes en los bajos, incluso lejos de la orilla y esparcidos. Estas elevaciones, en contraste con las más pequeñas, parecen estar hechas por la mano del hombre en combinación con el agua en el bajo, pero sólo existe aproximadamente uno por mecate. Hay bajos con sólo dos elevaciones de $2 \mathrm{~m}$ de longitud, $80 \mathrm{~cm}$ de ancho y $20 \mathrm{~cm}$ de altura, con sus hoyancos de hasta 3 o $4 \mathrm{~m}$ de longitud, $3 \mathrm{~m}$ de ancho y hasta 1.20 de profundidad. Donde hay numerosas elevaciones grandes, el terreno que las rodea presenta múl- tiples hoyancos. Aunque no puede sembrarse en los hoyancos puesto que se cubren de agua - por lo menos durante las épocas de lluvias-, sí se puede sembrar en los cuyos grandes asociados con fragmentos de pedernal. En contraste con la parte media del bajo, hay más cuyos chicos y grandes localizados a unos $25 \mathrm{~m}$ alrededor de las aguadas que se encuentran dentro de los bajos y de los arroyos fuera de sus bordes.

Además de una aguada de más de 5 ha de superficie, localizada en la orilla del gran bajo, Calakmul cuenta - como parte de su sistema hidráulico- con un canal de $250 \mathrm{~m}$ de longitud entre dos aguadas, cuya función es pasar agua de una aguada a otra, tal vez como lo haría el canal - mucho más largo- calificado por Puleston y Callender (1967) como defensivo, localizado entre Tikal y Uaxactún, y que podría haber pasado agua de un bajo a otro de oeste a este. Aparte de este canal, en Calakmul existe lo que pensábamos eran otros canales marcados por el follaje de los árboles y que son visibles desde la parte superior de la estructura I, que se extienden largamente al este de la estructura, salvo uno que corre hacia el sureste. También pensábamos que podría tratarse de antiguas veredas, como las localizadas en Costa Rica por medio de percepción remota, o bien de sacbés; esto último ha sido sugerido por Abel Morales L. (comunicación personal, 1993; véase Folan et al., 1995) y recientemente fue confirmado por May Hau en el campo (carta, 1995). Calakmul tiene además un arroyo modificado que puede clasificarse como un rasgo hidráulico lineal, pero no existe ninguna indicación de un campo drenado por el arroyo arriba mencionado. De 


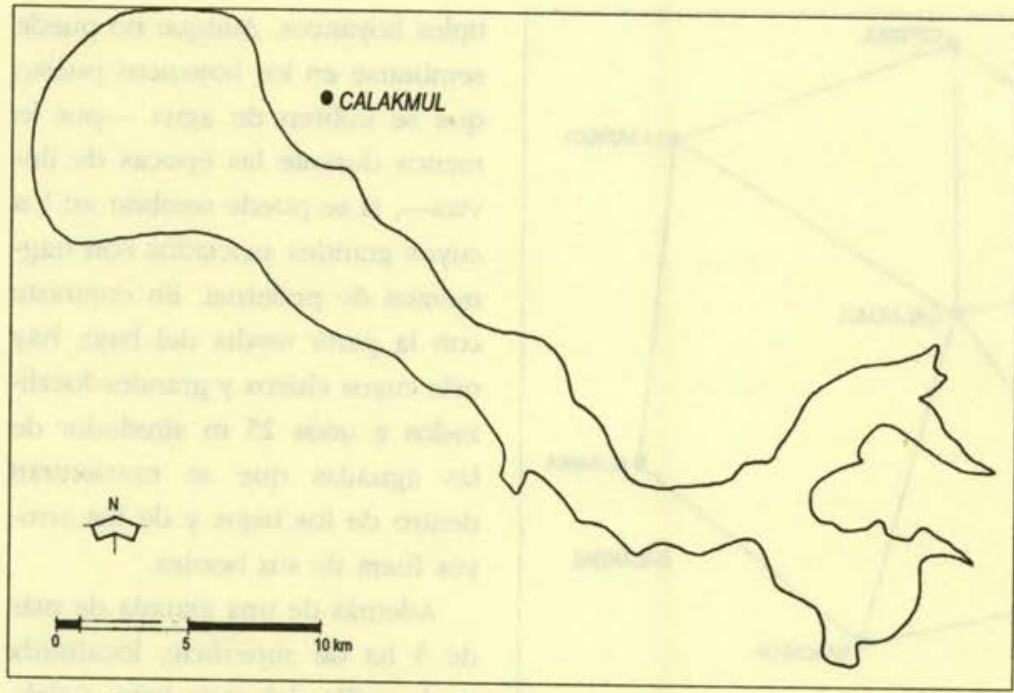

Figura 4. El bajo de El Laberinto (dibujo de Juan José Cosgaya Medina) vados dispersos. Sin embargo, ahora pensamos que representan, en realidad, el desperdicio del trabajo del pedernal que se realizó en los bajos, pues se excavaba para extraer la materia prima y se formaban así los hoyancos que se encontraron allí.

Estamos en condiciones de asegurar que las investigaciones llevadas a cabo por Domínguez Ca- acuerdo con Adams (1983), diremos que las orillas de los bajos podrian haber sido utilizadas a través del tiempo para la siembra, como también lo explicó Reynaldo Acevedo (comunicación personal, 1991) de Guatemala, cuya tesis de licenciatura aborda el tema de los bajos.

En contraste con las interpretaciones de Scarborough y Gallopin (1991) sobre Tikal, resultará de interés mencionar que aunque los investigadores de Tikal sugieren que las depresiones de forma irregular asociadas con el núcleo del gran centro urbano sirvieron como aguadas. También es interesante mencionar que la aguada más grande, localizada en el bajo junto a Calakmul, cuenta con una entrada y una salida que lleva el exceso de agua a otra aguada y sigue el arroyo hasta el centro del bajo sin dar - según los mapeadores- una indicación de que hubiera sido utilizada para el riego, como fue sugerido en el caso de Tikal por Scarborough y Gallopin (1991).

En determinado momento, los cuyos grandes, localizados al lado de los hoyancos en medio de los bajos, además de rodear las aguadas encontradas en los bajos, parecían campos eleEstudios de Cultura Maya. Vol. XXI, 2000

Instituto de Investigaciones Filológicas/

Centro de Estudios Mayas, UNAM ISSN: 0185-2574 rrasco (1993) alrededor de las habitaciones cercanas al bajo de El Laberinto registraron una gran cantidad de fragmentos de pedernal, mezclados con fragmentos de cerámica en su mayoría de tipo doméstico, lo que sugiere que alli podrían haber habitado técnicos dedicados a trabajar este material. Otros resultados de estas excavaciones son las muestras profundas del bajo, que servirán para determinar si éste constituía una laguna en el pasado, como se hizo en el periodo 1988-1989 en Calakmul.

Es muy probable que los antiguos mayas asentados alrededor de Calakmul sembraran en los espacios que quedaban entre sus casas, prefiriendo las orillas de los bajos para sembrar en el mes de mayo, y esperaran hasta octubre para decidir - basados en la primera cosecha- si sería recomendable sembrar por segunda vez en el bajo, entre los cuyos chicos y grandes. No hemos encontrado campos elevados en Calakmul; una posible excepción es una mención no comprobada de unos cuantos ubicados en el bajo localizado al este de la ciudad, drenado por el arroyo y que sale por detrás del bajo ubicado en la parte localizada más hacia el sureste 
del mapa, además de la posible existencia de campos elevados observados en una imagen de satélite que me facilitó R. E. W. Adams hace unos 10 años. Estas imágenes muestran diseños en forma de celosía que, según Adams, pueden representar canales y campos elevados con los que no nos hemos topado aún, a pesar de haber delineado mapas de bajos de unos $10 \mathrm{~km}^{2}$.

En caso de que existan campos elevados alrededor de Calakmul estos rasgos deberían estar visibles, si se les compara con los cuyos grandes y sus hoyancos que todavía están a la vista después de más de 500 años de existencia. De cualquier manera, podemos considerar tanto a los cuyos chicos como a los grandes como pequeños campos elevados para la horticultura en los bajos del Petén del norte, o protochinampas, diferentes de las que menciona Siemens. Al mismo tiempo se ignora cómo estos rasgos podrian haber mantenido sin hambre a un centro urbano de las dimensiones de Calakmul, aunque se trate de un asentamiento preindustrial, si no existian más cuyos pequeños por mecate en la época prehispánica, que resultaran suficientes para tener cerca de 625 matas de maíz por mecate, asociadas actualmente con las milpas halladas en las faldas de los bajos del Petén del norte.

Pese a que ahora sabemos un poco más sobre la práctica de la agricultura y la horticultura entre los mayas de Calakmul en la época mencionada, todavía falta mucho para saber a ciencia cierta cómo pudieron haber subsistido 40000 habitantes o más, dadas las condiciones prevalecientes en ese tiempo. Quizá fue por medio de técnicas agrícolas extensivas o intensivas, o por una combinación de ambas, sin descartar la posibilidad de la importación de granos desde lugares cercanos o lejanos, desarrollados en forma de terrazas (Eaton, 1976) o de campos elevados asociados con lugares como el río Candelaria (Siemens y Bulestan 1972) 1. XXI, 2000
De manera que, como se ha visto, ciertas secciones de los bajos servían por lo menos para la horticultura, lo que explica en parte por qué grandes ciudades como Calakmul, El Mirador y Tikal fueron construidas en las orillas de grandes bajos. En mi opinión, hasta no conocer profundamente el desarrollo de los mayas y su agricultura, a lo largo de diferentes regímenes climatológicos en Calakmul y otras zonas a través del tiempo, no comprenderemos con exactitud las causas que hicieron posible su esplendor y decadencia. Es decir que podemos relacionar este fenómeno con la posibilidad de que las aguas que se filtraban lateralmente bajo la tierra desde las elevaciones localizadas alrededor de los bajos servían para mantener húmedas las orillas de los bajos, donde Silverio Gallegos Osuna las ha visto salir durante la temporada de lluvias en el bajo de El Laberinto, formando así un sistema de riego natural (Gates y Folan, 1993; Gates y Folan, s.f.) que podría haber permitido hasta dos o tres cosechas anuales en periodos de precipitación adecuada, además de alimentar a las aguadas.

\section{Bibliografia}

AdAms R. E. W.

$1983^{\circ}$ "Ancient Land Use and Culture History in the Pasion River Region", en D. Z. Vogt y R. M. Leventhal, eds., Prebistoric Settlement Patterns, Essays in Honor of Gordon $R$. Willey, Albuquerque, University of New Mexico Press and Cambridge, Harvard University.

1986 Rio Azul Reports no. 2: The 1984 Season, San Antonio, University of Texas.

Coyoc Ramirez, M.

1989 "Los enterramientos humanos asociados a la estructura III de Calakmul, Campeche", ponencia presentada en el I Congreso Internacional de Mayistas. Homenaje a Alberto Ruz Lhuillier, 14 al 19 de agosto de 1989, San 
Cristóbal de Las Casas, Chiapas, México, Centro de Estudios Mayas, unam.

Domínguez Carrasco, M. R.

1993 "Calakmul, Campeche, y su sistema hidráulico", Los Investigadores de la Cultura Maya, 1, Universidad Autónoma de Campeche, Conacyt, México, pp. 42-46.

Dominguez Carrasco, M. R., y J. Gallegos Gómora

1989 Informe de trabajo del Proyecto Calakmul, 1984. Estructura VII, Información, 14: 56-84.

EATON, J. D.

1976 Ancient Agricultural Farmsteads in the Rio Bec Region of Yucatan, mimeo, San Antonio, University of Texas at San Antonio.

FLANNERY, K. V.

1972 "The Cultural Evolution of Civilizations", Annual Review of Ecology and Systematics, 3: 399-426.

Fletcher, L. A., J. May Hau, L. M. Florey folan y W. J. FOLAN

1987 Un análisis estadístico preliminar del patrón de asentamiento de Calakmul, Campeche, Universidad Autónoma del Sudeste, Campeche.

Folan, W. J.

1978 Proyecto Calakmul, proyecto enviado a la National Science Foundation, al National Geographic y al INAH (inédito).

1984 "El parque ecoarqueológico de Calakmul", Información, 8: 64-70.

1985a "Prefacio", en W. J. Folan (ed.), Contributions to the Arcbaeology and Etnohistory of Great Mesoamerica, Carbondale, Southern Illinois University Press.

1985b "Calakmul, Campeche: su centro urbano, estado y región en relación al concepto del resto de la gran Mesoamérica", Información, 9: $161-185$.

1988 "Calakmul, Campeche: el nacimiento de la tradición clásica en la gran Mesoamérica", Información, 13: 122-190.

Folan, W. J., J. Gunn, J. Eaton y R. Patch

1983 "Paleoclimatological Patterning in Southern Mesoamerica", Journal of Field Archaeology, 10 (4): $453-468$.

Folan, W. J., J. Marcus, S. Pincemin, M. R. Dominguez, L. Fletcher y A. Morales

1995a "Calakmul, Campeche: New Data from an Ancient Maya Capital in Campeche, Mexico", Latin American Anchaeology xxals 250 y 6.

Instituto de Investigaciones Filológicas/

Centro de Estudios Mayas, UNAM

ISSN: 0185-2574
Folan, W. J., J. Marcus y W. F. MILLeR

1995b "Verification of a Maya Settlement Model through Remote Sensing", Cambridge Archaeological Journal, 5 (2): 277-283.

Folan, W. J., Y Abel A. Morales

1996 "Calakmul, Campeche, México: La estructura II-H. Sus entierros y habitaciones", Revista Española de Antropología Americana.

Folan, W. J., J. May Hau, R. Counou Muñoz y R. GoNZÁL.EZ HEREDIA

1990 Calakmul, Campeche, México: su mapa. Una introducción, CIHS, UAc.

Folan, W. J., J. M. Garcia Ortega y M. C. Sánchez Gonzílez (coordinadores)

1992 Programa de Manejo de la Reserva de la Biósfera de Calakmul, Campeche, 4 vols., propuestas de programas de manejo, Cirs, UAC, Sedesol, Campeche, México.

Gates, Gary, Y W. J. Folan

s.f. "Hydrahulic Seepage and Bajo Edge Horticulture in Calakmul, Campeche", en preparación.

1993 "The Hydrogeologic Setting of the Aguadas in the Calakmul Biosphere Reserve, Campeche, Mexico", ponencia leída en la sesión "Paleoclimatología, paleohidrología y niveles del mar en América", XIII Congreso Internacional de Ciencias Antropológicas y Etnológicas, México, D. F., julio 29-agosto 5 de 1993.

GunN, J. D., y W. J. Folan

1995 "Tres ríos. Una superficie de impacto climático global interregional para las tierras bajas de los mayas del sureste. (La cuenca de los ríos Candelaria, Usumacinta y Champotón)", ponencia leída en el V Encuentro: Los Investigadores de la Cultura Maya, unc, 13-17 de noviembre de 1995.

Gunn, J. D., W. J. Folan y H. Robichaux

1994 "Análisis informativo sobre la descarga del sistema del río Candelaria en Campeche, México: Reflexiones acerca de los paleoclimas que afectaron a los antiguos sistemas mayas en los sitios de Calakmul y El Mirador", en W. J. Folan, coord., Campeche Maya Colonial, Colección Arqueología, UAc, Chiapas, México, pp. 174-197.

1995a "A Landscape Analysis of the Candelaria Watershed in Mexico: Insights into Paleoclimates Affecting Upland Horticulture in the 
Southern Yucatan Peninsula Semi-karst", Geoarchaeology: An International Journal, vol. 10, núm. 1, enero, pp. 3-42.

HANSEN, R.

1994 "Investigaciones arqueológicas en el norte del Petén de Guatemala. Una mirada diacrónica de los orígenes mayas", en W. J. Folan (coord.), Campeche Maya Colonial, unc, Campeche, pp. 14-51.

LAgunas, R. Z.

1985 "Exploración de la tumba 1 de la zona arqueológica de Calakmul, Campeche", Información, 9: 70-98.

LUNDELL, C. L.

1933 "Archaeological Discoveries in the Maya Area", Proceedings of the American Pbilosophical Society, vol. LXXII, núm. 3. Filadelfia, American Philosophical Society.

MARCUS, J.

1976 Emblem and State in the Classic Maya Lowlands: An Epigraphic Approach to Territorial Organization, Dumbarton Oaks, Washington, D. C.

1989 "Epigrafia de Calakmul, Campeche", ponencia presentada en el I Congreso Internacional de Mayistas. Homenaje a Alberto Ruz Lhuillier, 14 al 19 de agosto de 1989, San Cristóbal de Las Casas, Chiapas, México, Centro de Estudios Mayas, unam.

Morales L., A.

1994b "Las tumbas de dos personajes de la cultura maya", Deslinde, núms. 34 y 35 , pp. 5-6, Campeche, Camp.
NetTING, R. M.

1977 "Maya Subsistence: Mythologies, Analogies, Possibilities", en R. E. W. Adams (ed.), The Origins of Maya Civilization, University of New Mexico Press, Albuquerque.

Pérez Herrera, E.

1989 "Informe sobre los trabajos de conservación y restauración de los materiales arqueológicos de la tumba 1 de la estructura III de la zona arqueológica de Calakmul, Camp.", artículo aceptado para su publicación en Información, núm. 16.

Pincemin, S.

1989 Calakmul, Campeche. Informe de la temporada noviembre-diciembre de 1988, manuscrito, Campeche, Instituto Nacional de Antropología e Historia.

Puleston, D. E., y D. W. Callender Jr.

1967 "Defensive Earthworks at Tikal", Expedition, 9, núm. 3: 40-48.

Scarborough, V. L., y G. G. Gallopin

1991 "A Water Storage adaptation in the Maya Lowlands", Science, 251: 658-662.

SIEMENS, A.

1978 "Karst and the Prehispanic Maya in the Southern Lowlands", en P. Harrison y B. L. Turner II (eds.), Prebispanic Maya Agriculture, University of New Mexico Press, Albuquerque.

Siemens, A., y D. E. Puleston

1972 "Ridged Fields and Associated Features in Southern Campeche: New Perspectives in the Maya Lowlands", American Antiquity, 37: 228-239. 